\title{
The Feasibility of MR-Image Guided Prostate Biopsy Using Piezoceramic Motors Inside or Near to the Magnet Isocentre
}

\author{
Haytham Elhawary ${ }^{1}$, Aleksander Zivanovic ${ }^{1}$, Marc Rea ${ }^{1}$, Brian Davies ${ }^{1}$, \\ Collin Besant ${ }^{1}$, Donald McRobbie ${ }^{2}$, Nandita de Souza ${ }^{3}$, \\ Ian Young ${ }^{4}$, and Michael Lampérth ${ }^{1}$ \\ ${ }^{1}$ Mechanical Engineering Department, Imperial College London, South Kensington \\ Campus, SW7 2AZ, London, UK \\ \{h.elhawary, a.zivanovic, m.lamperth\}@imperial.ac.uk \\ ${ }^{2}$ Faculty of Medicine, Clinical Sciences Centre, Charing Cross Hospital, Imperial College \\ London, W6 8RF, London, UK \\ ${ }^{3}$ Cancer Research UK Clinical Magnetic Resonance Research Group, Institute of Cancer \\ Research, Royal Marsden NHS Foundation Trust, Downs Road, Sutton, Surrey, UK \\ ${ }^{4}$ Electrical Engineering Department, Imperial College London
}

\begin{abstract}
The excellent soft tissue contrast of Magnetic Resonance Imaging (MRI) has encouraged the development of MRI compatible systems capable of combining the advantages of robotic manipulators with high quality anatomical images. Continuing this development, a new five DOF prostate biopsy manipulator has been designed for use inside a closed 1.5T MRI scanner. Space constraints in the bore and the current trend to restrict field strength exposure for operators indicate that a master-slave configuration is ideal for controlling the robotic system from outside the bore. This system has been designed to work with piezoceramic motors and optical encoders placed inside or near the field of view of the scanner, using real time image guidance for targeting biopsies to specific lesions in the prostate. MRI tests have been performed to prove the feasibility of this concept and a one DOF proof-of-concept test rig implementing closed loop position control has been tested and is presented here. A first prototype of the slave manipulator has been designed and manufactured incorporating this new technology.
\end{abstract}

\section{Introduction}

\subsection{Prostate Cancer}

Each year over 27,000 men are diagnosed with prostate cancer in the UK, and it causes approximately 10,000 deaths [1]. Quick and reliable methods for diagnosis are therefore essential for selecting the right type of therapy, if any, to be used in each case. Currently, if a patient is suspected of having prostate cancer, a needle biopsy of the prostate is carried out using trans-rectal ultrasound (TRUS). This technique provides limited image resolution, giving rise to a possibility of diagnostic inaccuracies. 
Literature shows that for the sextant biopsy protocol, which is the standard in many centres, TRUS guidance leaves $15-31 \%$ of prostate cancers undetected [2]. The good soft tissue contrast of MRI makes it a more favourable means for the detection of lesions in the prostate. A robotic system which can perform accurate biopsies of the prostate under MRI guidance would eliminate many of the existing drawbacks of conventional prostate biopsy procedures.

\subsection{MRI Compatible Manipulators}

Over the past decade a number of MRI compatible manipulators have been developed [3-7]. The challenges in developing such devices are numerous. Ferromagnetic materials and electromagnetic actuators are prohibited, and noise from electronic circuits can considerably degrade the Signal to Noise Ratio (SNR) of the MR image. In addition, closed MRI scanners impose severe spatial constraints, providing poor access to the patient within the cylindrical bore of the scanner. There is also a growing trend to restrict the field strength to which medical practitioners are exposed [8]. This could give an important impulse to the development of tele-operated MRI compatible manipulators capable of being introduced into the scanner bore.

Two devices which perform MRI compatible prostate biopsy in a closed $1.5 \mathrm{~T}$ scanner have previously been developed [5,6], where positioning of the trans-rectal probe is performed by a passive manipulator in both cases. Long flexible rods which control the motion of the manipulator extend outside of the bore, and are actuated manually by the practitioner.

In this paper, a feasibility study is performed to demonstrate the viability of using piezoceramic motors placed inside or very near to the field of view of the MRI scanner to actuate a prostate biopsy manipulator. The motors will be used to position and fire a biopsy needle under real-time image guidance. The feasibility of this concept is demonstrated with a one DOF proof of concept prototype.

\section{System Design}

\subsection{System Requirements and Specifications}

The objectives of the system are to use MR image guidance to target abnormalities in the prostate and to perform a biopsy accurately and quickly. The system is designed for use inside a closed 1.5T MRI scanner, and will have a master-slave configuration. An endorectal probe with fiducial markers and an RF coil is inserted into the rectum of the patient, who is in the left lateral decubitus position. The probe is then attached to a slave robotic system that positions it, and fires a biopsy needle into a defined position in the prostate. The operator controls a master manipulator located inside the scanner room, and the slave system located inside the scanner bore, follows the movements of the master. Images from the MRI scanner are obtained in real-time and displayed to the practitioner so that the transrectal probe's position and any displacement of internal tissue can be seen at all times. A block diagram of the system is displayed in Fig. 1. 


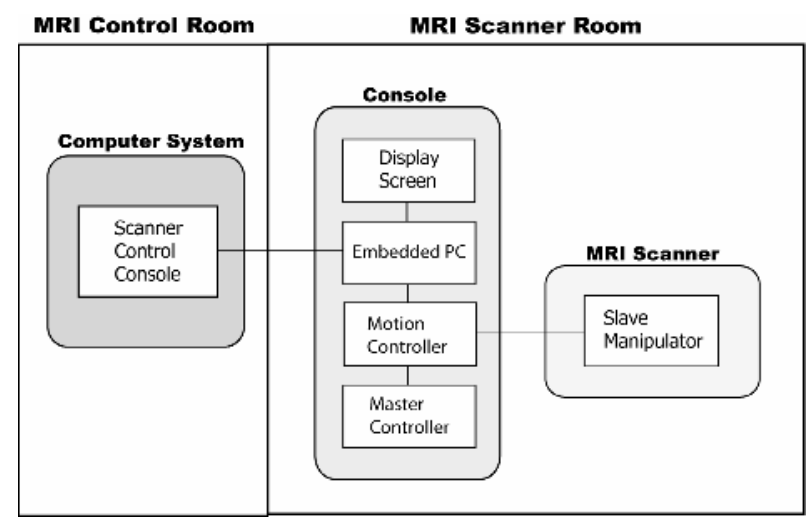

Fig. 1. Block diagram of MRI compatible master-slave prostate biopsy manipulator

To obtain the specifications of the manipulator, a number of conventional TRUS guided prostate biopsy procedures were observed. Movement of the endorectal probe in four degrees of freedom is necessary to reproduce the urologist's movements: three rotations (yaw, pitch and roll) centred on the anus and one translation (in/out). The ranges of motion of the slave manipulator are shown in Fig. 2 (a), together with a kinematic diagram Fig. 2 (b). In addition, a fifth DOF is required to position the biopsy needle (for $2-3 \mathrm{~cm}$ ) at the right depth before firing it into the prostate.

(a)

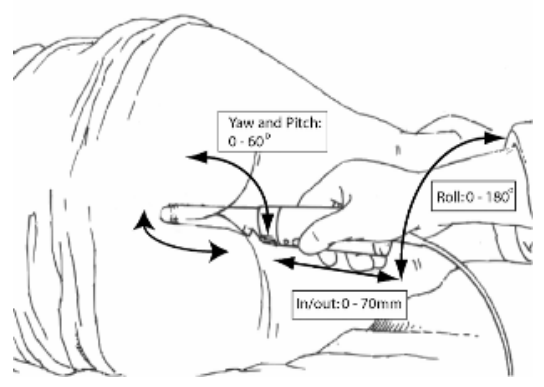

(b)

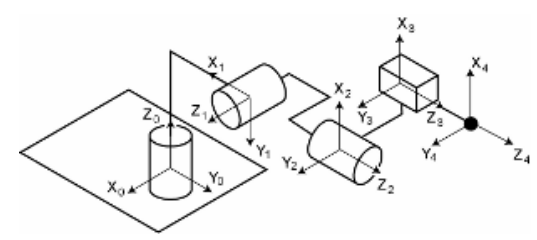

Fig. 2. (a) The four degrees of freedom required to replicate the prostate biopsy procedure, (b) a kinematic diagram of the system

\subsection{MRI Compatible Actuation Technologies}

In [9] four main categories of motion technologies were identified as suitable for use in MRI compatible manipulators: piezoceramic actuators, pneumatic and hydraulic systems and manual actuation. Piezoceramic motors were selected for use in this prostate biopsy manipulator, as they present precise positioning (in the order of nanometres) and virtually no backlash. They also have a hard brake which makes them rigid even during a power failure, which is an important safety measure for 
medical systems. More importantly, they are available in non-magnetic versions and are very compact.

Most of the manipulators that have used piezoceramic motors for actuation have generally followed one of two strategies: $(i)$ placing the motors inside the field of view of the scanner - due to noise from the motors interfering with the MR images, this approach requires turning the motors off during image acquisition [7], impeding real-time image guidance - and (ii) placing the motors at a distance from the imaging volume in the scanner, to avoid degradation of the Signal to Noise Ratio (SNR) and the creation of image artifacts [3,4]. A separation of around $1 \mathrm{~m}$ [4] usually proves enough distance to avoid deterioration of the SNR, but it also means that transmission mechanisms are needed. In [3] long titanium arms were used, while in [4] telescopic acrylic shafts transmitted the motion into the field of view of the scanner. One of the objectives for this system is to incorporate the motors inside or very near the field of view of the scanner, avoiding the need for a transmission mechanism. To achieve this, an alternative model of piezoceramic motors (Piezomotor PiezoLegs) was sourced, with a maximum force of approximately $7 \mathrm{~N}$, maximum speed of $12.5 \mathrm{~mm} / \mathrm{sec}$ and good MR compatibility. Small reflective surface mount optical encoders (Agilent AEDR-8300) were sourced and tested, to record the position of the slave.

(a)

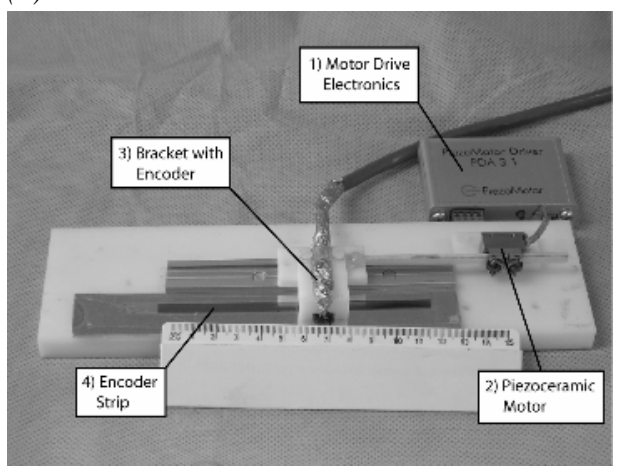

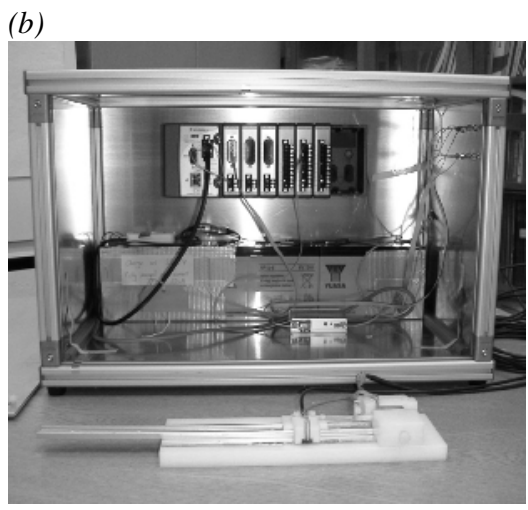

Fig. 3. (a) The one DOF prototype with a piezoceramic motor and optical encoder. (b) The shielded aluminium enclosure showing the motion controller, batteries and motor electronics.

\subsection{Feasibility Studies with a One DOF Test Rig}

To demonstrate the feasibility of placing the motors in the field of view of the scanner a one DOF test rig was designed and manufactured, as shown in Fig. 3(a). It included a piezoceramic motor (2) and an encoder (3), to implement closed-loop position control. The rig was constructed with Delrin ${ }^{\mathrm{TM}}$ and contained an aluminium rail. A motion controller (National Instruments Compact Rio 9004) with LabView was used to position the motor. For testing in the scanner, a shielded aluminium enclosure was constructed which housed the Compact Rio, a $24 \mathrm{~V}$ lead acid battery and the motor drive electronics. The enclosure was then connected to the scanner room's ground 
connection and placed 1-2m away from the scanner bore, acting as a Faraday cage. All wires that left the enclosure were shielded twisted pair cables, and were filtered with a low pass filter (TUSONIX 4209-053).

\section{MRI Tests and Results}

MRI compatibility tests were performed with a piezoceramic motor, an encoder and the one DOF test rig. Each component was placed on a fluid filled phantom and scanned in a 1.5T Siemens Magnetom Vision. The image sequences used to test the components were gradient echoes, which are more representative of the real-time nature of the image guided biopsy procedure for which the slave manipulator is designed. There are two principal types of image degradation: (i) an artifact that appears on the scan and (ii) a degradation of the SNR of the image. If an artifact was detected, then its size was quantified. This indicated how far a certain component had to be placed away from the region of interest to avoid unacceptable distortion of a desired area within the image. With respect to the SNR degradation, the SNR of an image of the component on a phantom was compared to the SNR of an image of the phantom without the component, to quantify the interference produced by the device. The definition of SNR used is the ratio between the mean signal in the $40 \times 40$ pixel region in the centre of the phantom on the image, and the standard deviation of the signal in the $40 \times 40$ pixel region at the bottom right hand corner of the image [3].

\subsection{Encoders and Motors}

The encoders were tested with a FLASH 2D gradient echo sequence (FOV=300mm, $\mathrm{TR} / \mathrm{TE}=200 / 10 \mathrm{~ms}, 256 \mathrm{x} 256, \mathrm{BW}=130 \mathrm{~Hz} /$ pixel, FA: 30, 9 slices, slice thickness and spacing: $5 \mathrm{~mm})$. A single encoder $(5.12 \times 3.96 \times 1.63 \mathrm{~mm})$ produced a localised image void enclosed in a rectangular region of $43 \times 16 \mathrm{~mm}$ in the transverse direction. Outside this region, the encoder has no effect on the image.

To test the variation of the SNR produced by the encoder, it was tested under various conditions (unwired, wired and powered) to see its effect on the image. The results for the slice located nearest to the centre of the field of view are presented in Table 1. The SNR of the images with the encoder are compared to the SNR of the phantom alone, and the reduction in SNR is calculated as a percentage. The results indicate that the reduction in SNR due to the encoder is virtually negligible.

Table 1. SNR variation of encoder under different conditions

\begin{tabular}{lll}
\hline Elements tested & SNR & SNR reduction (\%) \\
\hline Phantom & 138.9 & - \\
Phantom with encoder unwired & 138.3 & 0.4 \\
Phantom with encoder wired but not powered & 136.5 & 1.7 \\
Phantom with encoder powered & 135.4 & 2.6 \\
\hline
\end{tabular}

A non-magnetic linear actuator was tested on a phantom with a True Fisp gradient echo sequence $(\mathrm{FOV}=230 \mathrm{~mm}$, TR/TE: 6.46/3.05ms, 256x256, FA: 80 6 slices, slice 
thickness and spacing: $5 \mathrm{~mm})$. The motor $(22 \times 10.8 \times 18 \mathrm{~mm})$ produced its biggest artifact in the transverse direction spanning a rectangular region of $51 \times 21 \mathrm{~mm}$ represented in Fig. 4. Outside this region, the motor does not distort the image.

(a)

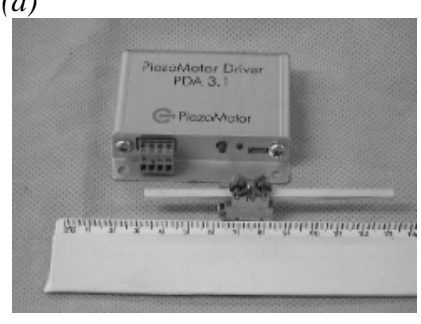

(b)

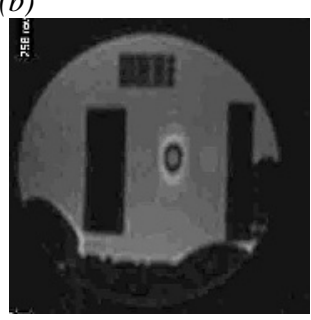

(c)

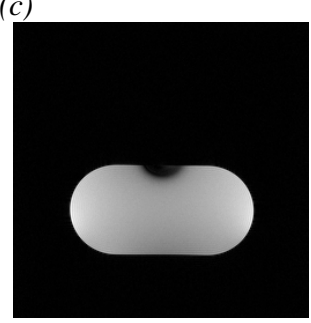

Fig. 4. (a) Piezomotor with drive electronics, (b) MR images of the phantom with the motor in coronal orientation and $(c)$ in transverse orientation

The motor was tested under various conditions to see its effect on the SNR, with the results for a slice located near to the field of view presented in Table 2 . The SNR reduction is under $14 \%$, which still makes the image perfectly usable for needle guidance.

Table 2. SNR variation of motor under different conditions

\begin{tabular}{lll}
\hline Elements tested & SNR & SNR reduction (\%) \\
\hline Phantom & 52.3 & - \\
Phantom with motor unwired & 48.7 & 6.9 \\
Phantom with motor wired but not powered & 48.3 & 7.6 \\
Phantom with motor powered but not actuated & 46.2 & 11.7 \\
Phantom with motor actuated at half max speed & 45.0 & 13.8 \\
Phantom with motor actuated at max speed & 45.1 & 13.7 \\
\hline
\end{tabular}

\subsection{One DOF Test Rig}

The one DOF test rig was placed on top of a phantom and introduced into the centre of the field of view, with closed loop position control implemented. Scans were taken using a True Fisp sequence $(\mathrm{FOV}=230 \mathrm{~mm}$, TR/TE $=6.46 / 3.05 \mathrm{~ms}, 256 \times 256$, FA: 80 , 10 slices, slice thickness and spacing: $5 \mathrm{~mm}$ ). The images only displayed small artifacts on certain slices (the test rig places the motor and encoder $20 \mathrm{~mm}$ away from the phantom). The SNR was tested under various conditions with the results for the slice nearest to the field of view presented in Table 3. The SNR degradation is greater than that of the motor and encoder considered separately, possibly due to the fact that the encoder produces pulses as it passes the lines on the encoder strip, which have some harmonics that can interfere with the sensitive RF coils of the scanner. This effect can be reduced by increasing the attenuation of the filters in the shielded enclosure at the Larmor frequency of the scanner. This feature is currently being implemented and this should result in a reduction of noise levels. In addition, the motors and encoders on 
the slave manipulator will be located several $\mathrm{cm}$ away from the main region of interest, which should also greatly reduce the noise interference produced.

Table 3. SNR variation of 1 DOF testrig under different conditions

\begin{tabular}{lll}
\hline Elements tested & SNR & SNR reduction (\%) \\
\hline Phantom & 63.2 & - \\
Phantom with test rig wired but not powered & 61.8 & 2.2 \\
Phantom with test rig powered & 46.3 & 26.7 \\
Phantom with test rig with position control & 45.7 & 27.6 \\
\hline
\end{tabular}

\section{Hardware Developments}

Once the feasibility of implementing closed loop position control with real time image guidance had been demonstrated, a first prototype of the slave manipulator incorporating this technology was built. This prototype incorporates only 2 DOF in an $\mathrm{XY}$ stage and is shown in Fig. 5.

(a)

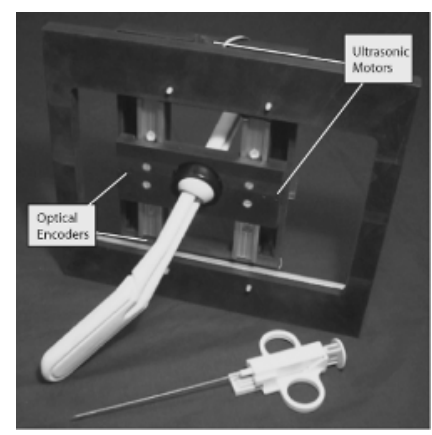

(b)

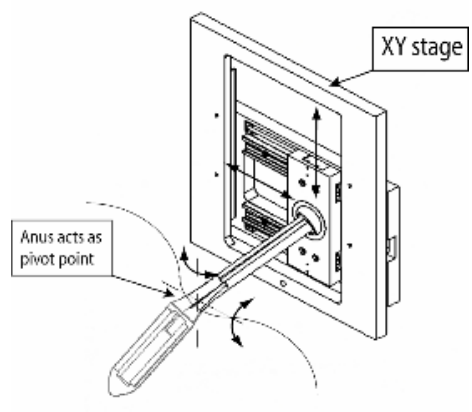

Fig. 5. Slave manipulator with endorectal probe and MRI compatible biopsy needle. The piezoceramic motors and encoders are incorporated into the structure.

An endorectal probe is placed into the anus, with the sphincter acting as a pivot point. The slave, which is made of glass filled Nylon, moves the other extreme of the probe (which is hinged via a plastic spherical bearing) varying the pitch and yaw angles. Each axis contains two piezoceramic motors which are embedded in the structure, two aluminium rails to provide smooth linear motion and an optical encoder. The slave is attached to the scanner table via a positioning arm.

Tests in the MRI scanner have proven that this manipulator is compatible, although rigorous tests to quantify the impact on SNR are still pending. Further design is underway to incorporate the other three DOF: probe translation, roll and needle depth insertion. Work is ongoing to interface the master console, the graphical user interface of the system and the MRI scanner to provide a fully integrated system. Target accuracy must also be assessed on phantoms before moving onto clinical trials. 


\section{Conclusions}

The master-slave prostate biopsy system described in this paper intends to introduce piezoceramic motors into the field of view of the scanner, eliminating the need for a transmission mechanism. Feasibility tests have been performed with a one DOF test rig to see if this objective is viable, yielding positive results. The test rig, which included an encoder and a piezoceramic motor, implemented closed loop position control with a reduction of under $28 \%$ of the SNR and $36 \mu \mathrm{m}$ accuracy. This renders images with sufficient quality for MR image guided biopsy. The power source and electronics for the system were housed in an aluminium shielded enclosure placed just outside the entrance of the scanner bore, and all cables exiting the enclosure were filtered. The noise can be further reduced by improving the attenuation of these filters and tests are currently underway to demonstrate this. A first prototype of the slave manipulator has been designed and manufactured which includes 2 of the 5 DOF required.

\section{References}

1. Cancer Research UK, Men's Cancers Factsheet - June 2004, World Wide Web: http://info.cancerresearchuk.org/images/publicationspdfs/factsheet_men_June2004 .pdf

2. D'Amico AV, Tempany CM, Cormack R, Hata N, Jinzaki M, Tuncali K, et al. Transperineal magnetic resonance image guided prostate biopsy. J Urol 2000;164(2): pp. 385-7.

3. Chinzei K, Hata N, Jolesz FA, Kikinis R. Surgical Assist Robot for the Active Navigation in the Intraoperative MRI: Hardware Design Issues. In: Proc. 2000 IEEE/RSJ International Conf Intelligent Robots and Systems; 2000; Maui, HI, USA: 1. pp. 727-732.

4. Larson BT, Erdman AG, Tsekos NV, Yacoub E, Tsekos PV, Koutlas IG. Design of an MRI-Compatible Robotic Stereotactic Device for Minimally Invasive Interventions in the Breast. Journal of Biomechanical Engineering - Transactions of the ASME 2004;126(4): pp. 458-465.

5. Beyersdorff D, Winkel A, Hamm B, Lenk S, Loening SA, Taupitz M. MR imaging-guided prostate biopsy with a closed MR unit at $1.5 \mathrm{~T}$ : initial results. Radiology 2005;234(2): pp. 576-81. Epub 2004 Dec 22.

6. Fichtinger G, Krieger A, Susil RC, Menard C, Coleman JA, Atalar E, et al. Transrectal Prostate Biopsy Inside Closed MRI Scanner with Remote Actuation, under Real-Time Image Guidance. In: Medical Image Computing and Computer-Assisted Intervention; 2002; Tokyo, Japan: Lecture Notes of Computer Science 2488, Part 1. pp. 91 - 98.

7. Masamune K, Kobayashi E, Masutani Y, Suzuki M, Dohi T, Iseki H, et al. Development of an MRI-compatible needle insertion manipulator for stereotactic neurosurgery. Journal of Image Guided Surgery, Copyright (C) 1995;1(4): pp. 242 - 248.

8. Directive 2004/40/EC of the European Parliament and of the council of 29 April 2004 on the minimum health and safety requirements regarding the exposure of workers to the risks arising from physical agents (electromagnetic fields),

9. Elhawary H, Zivanovic A, Davies B, Lamperth M. A Review of Magnetic Resonance Imaging Compatible Manipulators in Surgery. Proceedings of the Institution of Mechanical Engineers, Part H: Journal of Engineering in Medicine 2006;220(3): pp. 413-424. 